\title{
Special Issue on Reconfigurable Robots
}

\author{
Jinguo Liư ${ }^{1 *}$ Y Yuwang Liu' and Guangbo Hao ${ }^{2}$
}

Reconfigurable robots have the characteristics that structures can be changed or reorganized. They can adapt to different environments and tasks through changing configurations or structures, which is a hot research direction in recent years. Existing researches has not yet formed a complete theoretical method system of reconfigurable robots. Therefore, exploring new design theories, key technologies and typical applications is an effective way to promote the rapid development of this field.

We received 20 papers in this special issue and there are 8 papers selected for publication, covering several aspects such as reconfigurable theory and configuration synthesis, control algorithm, reconfigurable connecting mechanism (RCM), and technical application.

First, there is a need of configuration synthesis theory to guide reconfigurable robot research. As a type of multi-configuration mechanism that can operate when under-actuated, metamorphic mechanisms were proposed more than two decades ago and generated significant interest. Yang and his colleagues researched the structural synthesis of source mechanisms for metamorphic mechanisms. The equivalent resistance matrix of the deformation joint is proposed according to the equivalent resistance gradient model and the constraint state matrix. By deriving the constraint form vector of the deformation joint, the structure synthesis matrix of the deformation mechanism is obtained from the equivalent resistance matrix. On this basis, a kinematic diagram synthesis of the source metamorphic mechanism of the planar single-loop metamorphic mechanism is proposed which is based only on the 14 one or zero degree-of-freedom linkage groups. Li and his colleagues proposed a new reconfigurable joint based on the Rubik cube, named rA. Joint

\footnotetext{
*Correspondence: liujinguo@sia.cn

${ }^{1}$ State Key Laboratory of Robotics, Shenyang Institute of Automation,

Chinese Academy of Science, Shenyang 110016, China

Full list of author information is available at the end of the article
}

rA is used to construct metamorphic parallel mechanisms with topological structures and mobility changes. 36 kinds of limb phases and 56 parallel mechanism configurations are enumerated using screw theory.

Second, the control of reconfigurable modules has always been a hot issue of research. Chen et al. proposed a comprehensive optimization method to solve obstacle avoidance path planning and discrete trajectory tracking of a super-redundant manipulator. Path planning is established on the Bezier curve, and the control points of the Bezier curve are adjusted by the particle swarm algorithm.

With the development of intelligent flexible manufacturing, traditional industrial manipulators with a single configuration cannot meet multiple tasks. Reconfigurable robots have developed rapidly which could change their configurations and end effectors for different tasks. The $\mathrm{RCM}$ is the core component of reconfigurable robots. $\mathrm{Xu}$ et al. developed two intelligent modular RCMs with light weight, high load, and large pose error tolerance. One is driven by shape memory alloy and recovery spring. The other is driven by an electromagnetic coil and locked by a permanent magnet and key. The RCMs were integrated into actual robots and experiments were carried out.

Furthermore, reconfigurable mechanisms have also been widely used in civil, aerospace and other fields. Zhao and his colleagues proposed a new method for the surface design of the space reflector antenna based on the polar scissor unit. A new closed-loop deployable structure is designed combining plane polar scissor unit and space polar scissor unit, called Polar Scissor Deployable Antenna (PSDA). The over-constraint problem is solved by releasing the curve constraint at the end of the plane scissors mechanism. The results show that the design method can effectively fit the rotating surface, which provides a new idea for the construction of large space reflector antennas. Zhao and his colleagues studied the design of deployable kinematic chains (DKCs) to 
improve the storage performance of multi-arm robots. A platform strategy for storing DKCs and a comprehensive assembly method of multi-arm robot mechanism is proposed, which is formed by connecting a multi-arm robot mechanism and an operating object in a parallel mechanism. Two multi-arm robot mechanisms with four DKCs are proposed based on this method. Wei and his colleagues proposed a wheel-leg mobile platform with two motion modes based on a closed-chain mechanism. The speed capability and maneuverability of the closedchain mechanism are improved using kinematic analysis and parameter optimization. The performance of the two motion modes is analyzed and compared through dynamic simulation and experimental verification. Zhang et al. reported on the design, analysis and experiment of a variable stiffness passive compliant device composed of a reconfigurable elastic inner skeleton and an origami shell. The reconfigurable skeleton is composed of two elastic trapezoid four-bar linkages arranged in orthogonal. The stiffness change is produced by the passive deflection of the elastic limbs, which can be realized quickly and simply by actively switching the arrangement of the leaf spring and the passive joints.

We are fully aware of the on-going research and development efforts on reconfigurable robot research community and industry. It is hoped that the selected papers of the special issue can provide some foresight for future research and development, and a good reference for industry practices.

\section{Acknowledgements}

Not applicable.

\section{Authors' Information}

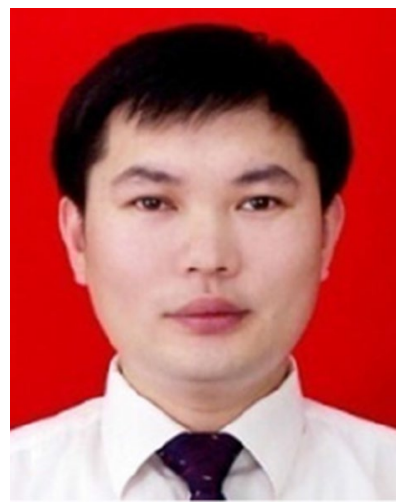

Jinguo Liu received his PhD degrees in Mechatronics from Shenyang Institute of Automation (SIA), Chinese Academy of Sciences (CAS) in 2007. Since January 2011, he has been a Full Professor with SIA, CAS. He is also holding the Assistant Director position of State Key Laboratory of Robotics from 2008 and the Associate Director position of Center for Space Automation Technologies and Systems from 2015. His research interests include bio-inspired robotics and space robot. He has authored/coauthored three books, over one hundred papers and fifty patents in above areas. He is a senior member of the IEEE, IEEE
Technical Committee on Safety, Security, and Rescue Robotics, IEEE Technical Committee on Marine Robotics, and the Senior Member of Chinese Mechanical Engineering Society. He services as the Associate Editor or Technical Editor of several journals such as IEEE/ASME Transactions on Mechatronics, Mechanical Sciences, Science China Technological Sciences, Chinese Journal of Mechanical Engineering, and Chinese Journal of Aeronautics.

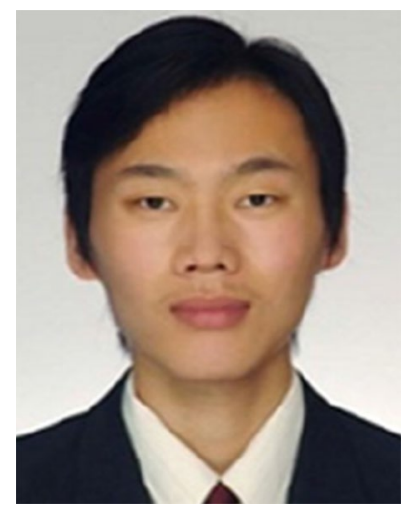

Yuwang Liu is a full Professor of Mechatronic Engineering. He received the PhD degree in Robotics from Shenyang Institute of Automation Chinese, Academy of Sciences in 2010, and then has been working here. His research interest include soft robot modelling, cooperative robot system, variable stiffness joint design. He has authored/coauthored over 45 papers including top journals, published 2 monographs, and applied for 67 invention patents. Professor Liu has won the first prize of Liaoning Technical Invention and the second prize of Liaoning Natural Science Academic Achievement. He has been selected as the Liaoning Top Young Talents, and the Cross Innovation Group of Chinese Academy of Sciences.

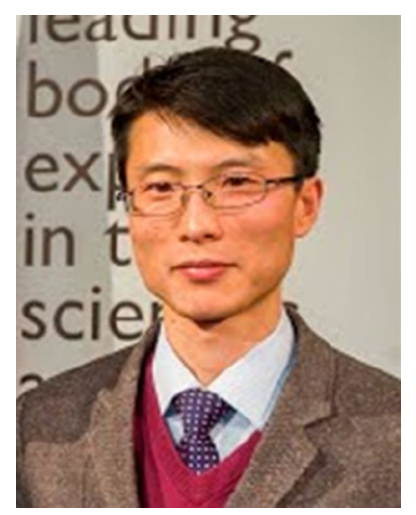

Guangbo Hao is a Senior Lecturer with University College Cork, Ireland. His research areas lie in design of compliant mechanisms and robotics and their applications in precision engineering, energy harvesting and biomedical devices. He obtained his PhD degree in Mechanical Engineering from HeriotWatt University in 2011. He is a member of ASME and an elected member of the ASME Mechanisms and Robotics Committee. He is serving as the Editorin-Chief of the IFToMM affiliated journal: Mechanical Sciences and the Associate Editor of ASME Journal of Mechanisms and Robotics. He has won some accolades including the 2017 and 2018 ASME Compliant Mechanisms Awards in a row. He has published over 130 peer-reviewed journal papers.

\section{Funding}

Not applicable.

\section{Competing Interests}

The authors declare no competing financial interests. 


\section{Author Details}

1 State Key Laboratory of Robotics, Shenyang Institute of Automation, Chinese Academy of Science, Shenyang 110016, China. ${ }^{2}$ School of Engineering, University College Cork, Cork, Ireland.

Received: 16 October 2020 Accepted: 28 October 2020

Published online: 20 November 2020

\section{Publisher's Note}

Springer Nature remains neutral with regard to jurisdictional claims in published maps and institutional affiliations.
Submit your manuscript to a SpringerOpen ${ }^{\circ}$ journal and benefit from:

- Convenient online submission

- Rigorous peer review

- Open access: articles freely available online

- High visibility within the field

- Retaining the copyright to your article

Submit your next manuscript at $\boldsymbol{\nabla}$ springeropen.com 